\title{
LA INCIDENCIA DE LOS VALORES CULTURALES EN EL VOLUNTARIADO: EL CASO DE EUROPA
}

\author{
POR \\ Antonio ARIZA MONTES, \\ Vicente FERNÁNDEZ RODRÍGUEZ y \\ Pilar TIRADO VALENCIA ${ }^{1}$
}

\section{RESUMEN}

La realidad del voluntariado es sumamente compleja hasta el punto de que resulta complicado definir y caracterizar el trabajo voluntario, dada la gran variedad de interpretaciones, motivaciones, variables sociodemográficas y aspectos culturales que configuran el perfil de los voluntarios. El objetivo de este trabajo es analizar la influencia conjunta de algunas variables sociodemográficas, así como de los valores culturales de índole secular o tradicional, sobre el perfil de los voluntarios en Europa. Además, se investiga qué variables orientan a los voluntarios hacia un determinado tipo de voluntariado u otro. Para ello se ha aplicado principalmente una metodología de regresión logística a partir de la información disponible en la European Value Study. Los resultados obtenidos ayudan a establecer una caracterización del voluntariado en Europa, y confirman la influencia de los valores culturales, en primer lugar, en la realización o no de trabajos de voluntariado, y en segundo lugar, en la elección que hacen estas personas del tipo de actividad con la que están comprometidos. Al analizar dos tipos de voluntariado de motivación supuestamente muy diferente, se concluye que existe un grupo de valores que influyen en ambos, aunque el sentido y la intensidad en la que lo hacen sea diferente; por otra parte, algunos valores tienen influencia o no en la realización de trabajos de voluntariado, dependiendo del tipo específico al que nos refiramos.

Palabras clave: valores sociales, altruismo, organizaciones no lucrativas, trabajo no retribuido, tipos de voluntariado.

\footnotetext{
${ }^{1}$ Universidad Loyola Andalucía. Direcciones de correo electrónico: ariza@uloyola.es, vicentef@uloyola.es y ptirado@uloyola.es.

REVESCO Nº 120 - Primer Cuatrimestre 2016 - ISSN: 1885-8031 - www.ucm.es/info/revesco

http://dx.doi.org/10.5209/rev_REVE.2016.v120.49154
}

Fecha de recepción: 17/10/2014

Fecha de aceptación: 09/04/2015 
Claves ECONLIT: A13, D64, L31

THE INFLUENCE OF CULTURAL VALUES OVER VOLUNTEERING: THE CASE OF EUROPE

\begin{abstract}
It is very difficult to define and typify volunteering due to the huge complexity voluntary work phenomenon has, including a large variety of interpretations, motivations, socio-demographic variables and cultural aspects associated to volunteers profile. The main objective of this study is to analyze the combined influence of some socio-demographic variables and cultural values, secular or traditional ones, over volunteers profile in Europe. Additionally, the study investigates what variables guide volunteers towards each volunteering type. A logistic regression methodology has been applied to the information obtained from the European Value Study. The results help to establish a characterization of volunteering in Europe, and confirm the influence of cultural values, on one hand, over carrying out or not voluntary work, and on the other hand, the influence over volunteers' choice of the type of activity to be committed to. Analyzing two volunteering types with supposedly very different motivations, we come to the conclusion that a group of cultural values influence both of them, although in different direction and intensity; at the same time, some values have influence or not over volunteering depending on the specific type.
\end{abstract}

Keywords: social values, altruism, nonprofit organizations, nonremunerated work, types of volunteering.

\title{
1. INTRODUCCIÓN
}

Los niveles de participación e implicación de los individuos en las actividades de voluntariado constituyen un indicador de la capacidad de una sociedad para identificar cuáles son sus problemas más acuciantes, así como de su compromiso con la búsqueda de soluciones. Sin embargo, la realidad del voluntariado es sumamente compleja, hasta el punto de que resulta complicado definir y caracterizar el trabajo voluntario, dada la gran variedad de interpretaciones, motivaciones, variables sociodemográficas y aspectos culturales que configuran el perfil de los voluntarios, analizados tanto a nivel individual (persona a persona), como en términos colectivos (asociaciones y redes de solidaridad). 
El conocimiento de este perfil resulta interesante para los gestores de entidades no lucrativas a la hora de articular estrategias de captación de personal, organización y asignación de tareas, búsqueda de fondos, o de diseñar políticas orientadas a mejorar la satisfacción de los voluntarios y, por lo tanto, su nivel de compromiso con la actividad que realizan y con la organización que les da soporte. Diferentes investigaciones ponen de manifiesto que existen variables sociodemográficas y contextuales que inciden de manera desigual sobre el perfil del voluntariado, según países o niveles de desarrollo. Además, los resultados de los estudios desarrollados en esta materia ofrecen conclusiones dispares según el momento en el que se realicen las investigaciones, ya que el entorno sociocultural evoluciona con el tiempo, lo que evidencia que el voluntariado presenta perfiles dinámicos que están permanentemente en proceso de cambio.

El análisis de la influencia que ejercen los valores sobre el perfil del voluntariado ha sido otra cuestión ampliamente abordada desde diferentes disciplinas como la sociología, la psicología, la economía, la gestión de recursos humanos o la organización empresarial, entre otras, sin llegar a resultados concluyentes en esta materia (Inglehart, 1997; Hofstede, 2001; Grönlund y otros, 2011).

Si las razones que explican el por qué realizar o no tareas de voluntariado constituyen ya de por sí un objeto de investigación, la elección de dónde desarrollar esta actividad es un asunto tan enigmático o más si cabe, ya que no resulta sencillo predecir las preferencias de los voluntarios sobre el tipo de voluntariado al que dedicar su tiempo de manera altruista. En este sentido habría que preguntarse si el voluntariado constituye un hecho en sí mismo o está alineado con un tipo de actividad concreta: ecológico, humanitario, religioso, político, sindical... La pregunta que cabría hacerse en este punto es si el sustantivo prima sobre todo lo demás o, por el contrario, tal sustantivo sólo adquiere significado cuando se acompaña de un adjetivo concreto, que necesariamente estará condicionado por los valores y creencias personales del individuo.

Asumiendo la dificultad que entraña llevar a cabo una caracterización de una realidad tan compleja como la del voluntariado, el presente estudio se plantea dos objetivos básicos. En primer lugar, analizar la influencia conjunta de algunas variables sociodemográficas y contextuales, así como de los valores culturales de índole secular o tradicional, sobre la propensión a dedicarse a tareas de voluntariado en Europa. En segundo lugar, investigar si 
determinados valores orientan a los voluntarios hacia un determinado tipo de voluntariado u otro.

Este trabajo se estructura del siguiente modo: tras la introducción inicial, en el segundo epígrafe se justifica el interés del estudio, se presentan las variables incorporadas en el modelo propuesto, y se plantea el objetivo general de la investigación así como sus hipótesis. A continuación, en el tercer apartado, se describe la metodología empleada y seguidamente, en el cuarto, se analizan los resultados obtenidos. El artículo finaliza con la discusión y la exposición de las conclusiones más relevantes.

\section{JUSTIFICACIÓN}

La bibliografía relativa a esta materia identifica varias categorías de factores relacionados con el voluntariado: características personales, estatus familiar y variables demográficas, situación socioeconómica e implicación con la comunidad, entre otros (por ejemplo, House, 1981; Smith 1994; Brehm y Rahn, 1997; Guterbock y Fries, 1997; Hall y otros, 1998; Independent Sector, 1999; Putnam, 2000; Hall, McKeown y Roberts, 2001; Voicu y Voicu, 2009). Tradicionalmente estos factores se han venido agrupando en dos niveles de análisis: variables sociodemográficas de índole familiar y personal (edad, género, estatus familiar, nivel de ingresos, cualificación), y variables contextuales (situación laboral, características del trabajo, población, etc.). Estos elementos configuran el capital humano, el capital social y el capital cultural del voluntario, cuya influencia sobre la actividad del voluntariado ha sido puesta de manifiesto en numerosos estudios previos (por ejemplo, Wilson y Musick, 1997; Brown y Ferris, 2007; Wang y Graddy, 2008).

Sin embargo, una línea de investigación más reciente considera que éstas no son las únicas variables que determinan el voluntariado. Las creencias y los valores implícitos en la cultura también se asocian con este fenómeno (Musick y otros, 2000; Plagnol y Huppert, 2010). Desde este paradigma, la evolución de los valores culturales en las sociedades modernas, influidos no sólo por el papel que juegan en ellas las tradiciones religiosas, sino también por los cambios sociales, así como por su desarrollo económico y tecnológico, ha sido investigada en profundidad en los influyentes trabajos de Inglehart (1997), Inglehart y Baker (2000), Inglehart (2003) e Inglehart y Welzel (2005). En este sentido, Inglehart (2003) defiende que el voluntariado está relacionado con el grado de industrialización de un país, de tal modo que el progreso de las naciones desde la economía agraria, donde predominan los 
valores de corte tradicional, hacia una economía industrial en la que prevalecen valores de sustrato más secular-racional, provoca un aumento de la participación en actividades de voluntariado.

La dicotomía entre valores seculares y tradicionales emerge de una concepción diferente de las sociedades desarrolladas frente a las no desarrolladas en lo que respecta a sus normas y creencias políticas, sociales y religiosas, asumiendo así que el desarrollo económico ejerce una influencia notable sobre los valores culturales. La investigación sobre la modernización y su efecto en los cambios culturales ha generado dos escuelas de pensamiento. La primera enfatiza la convergencia de los valores culturales hacia la secularización como resultado de la modernización, anticipando la pérdida de protagonismo de los valores tradicionales y su reemplazo por valores seculares (Bradshaw y Wallace, 1996). La segunda mantiene la persistencia de los valores tradicionales a pesar de los cambios económicos y políticos, y defiende que estos valores son independientes de las condiciones del entorno, manteniéndose al margen de la influencia de los cambios culturales originados por el desarrollo económico (DiMaggio, 1994) ${ }^{2}$.

Las características sociodemográficas y contextuales que definen el perfil de los voluntarios, así como la incidencia de los valores culturales sobre el voluntariado, han sido objeto de estudio en diferentes investigaciones obteniéndose resultados no siempre coincidentes. Algunos de los resultados de estos trabajos se resumen a continuación. La falta de consenso sobre las variables y los valores que determinan el perfil de los voluntarios, así como sobre los factores que influyen en sus preferencias a la hora de elegir entre distintos tipos de trabajo voluntario, justifican la oportunidad de este estudio.

\section{FACTORES SOCIODEMOGRÁFICOS Y CONTEXTUALES}

\section{A. Voluntariado: Género y roles}

Algunos estudios demuestran que no hay diferencias en el nivel de compromiso de los voluntarios que puedan explicarse por razones de género (Hodgkinson y Weitzman, 1996), aunque sí podrían encontrarse patrones distintos cuando esta variable se analiza conjuntamente con la edad; entre los jóvenes, las mujeres tienden a dedicar más tiempo al voluntariado que los hombres (Wuthnow, 1995; Einolf, 2011), mientras que entre los voluntarios de más edad, el patrón se invierte (Gallagher, 1994). La razón por la que estas

\footnotetext{
${ }^{2}$ Para profundizar en este debate se puede consultar el trabajo de Inglehart y Baker (2000).
} 
circunstancias no provocan mayores diferencias entre los dos colectivos podría interpretarse en términos de disponibilidad o falta de disponibilidad de tiempo (Gallagher, 1994; Rosenthal y otros, 1998).

\section{B. ¿Existe una edad ideal para el voluntariado?}

Los resultados de los posibles vínculos entre la edad y el voluntariado tampoco son concluyentes. Algunos autores establecen una relación positiva (Cappellari y Turati, 2004; Choi y DiNitto, 2012), mientras que otros estudios determinan que la tasa de voluntariado tiende a disminuir durante la transición de adolescente a adulto, y alcanza el máximo entre una edad media y la madurez (Schoenberg, 1980; Menchik y Weisbrod, 1987; Herzog y otros, 1989; National Association of Secretaries of State, 1999). Sin embargo, la investigación sobre la realidad canadiense realizada por Gómez y Guntherson (2003) demuestra un mayor grado de voluntariado entre los jóvenes de 15 a 19 años, coincidiendo con el periodo de menor actividad laboral.

\section{Formación del voluntario}

En este caso, los estudios empíricos si son bastante concluyentes acerca de la incidencia, a nivel individual, del nivel de formación sobre el ejercicio de actividades de voluntariado. De hecho, este factor es considerado por algunos autores como el mejor predictor del voluntariado (Mesch y otros, 2002; Gómez y Guntherson, 2003; Grønbjerg y Never, 2004; Gesthuizen y Scheepers, 2012). Más recientemente, Van Ingen y Dekker (2011) concluyen que el papel de la educación también ha evolucionado, diluyendo la influencia que ejercía esta variable sobre la participación en actividades de voluntariado.

Los autores que defienden la relación formación-voluntariado la interpretan en un doble sentido. Por un lado, desde una perspectiva psicológica, los estudios superiores podrían tener un impacto positivo en el grado de concienciación de los problemas sociales, en una empatía creciente, o en el desarrollo de la autoconfianza (Brady y otros, 1995; Rosenthal y otros, 1998); por otra parte, desde un punto de vista contextual, las personas con mayor formación podrían estar más dispuesta a colaborar como voluntarios (Brady y otros, 1999) y a pertenecer a más organizaciones (Herzog y Morgan, 1993). 


\section{Nivel económico del voluntario}

Algunos autores han demostrado que los individuos con un nivel socioeconómico alto (medido a través de la educación y el nivel de ingresos) presentan una mayor predisposición hacia el voluntariado, frente a los de nivel socioeconómico bajo (Vaillancourt, 1994; Guterbock y Fries, 1997; Hall y otros, 1998; O'Neill y Roberts, 1999; Hall, McKeown y Roberts, 2001). Si bien es cierto que las horas de voluntariado están relacionadas inversamente con el nivel retributivo del individuo, ya que los costes de oportunidad son mucho mayores (Wolf y otros, 1993).

Al mismo tiempo, Freeman (1997) mantiene que existe una relación negativa entre ingresos salariales y voluntariado, mientras que otros autores concluyen que esa relación es positiva sólo para determinados colectivos: entre los adultos solteros (Mesch y otros, 2002; Segal, 1993), y para determinados tipos de voluntariado, en concreto en el caso de las actividades relacionadas con la educación y la salud (Raskoff y Sundeen, 1995), pero sin embargo, no tiene ningún impacto sobre el de carácter religioso.

\section{E. Status familiar del voluntario}

Ciertos estudios confirman que existe alguna correlación entre la dedicación al voluntariado y el estado civil (Menchik y Weisbrod, 1987; Vaillancourt, 1994; Day y Devlin, 1996; Mesch y otros, 2002; Nesbit, 2012). En este sentido, Menchik y Weisbrod (1987) encuentran una mayor tasa de voluntariado entre las personas casadas, mientras que Vaillancourt (1994) defiende que las mujeres solteras hacen más trabajo de voluntariado que las casadas.

Por otra parte, Menchik y Weisbrod (1987) asocian el incremento del voluntariado con la presencia de hijos en el hogar; este hecho también es confirmado por Vaillancourt (1994) cuando estos tienen entre 3 y 15 años. En la misma línea, la investigación realizada por Gómez y Guntherson (2003) da más valor a la presencia de niños en casa que al estado civil. Por su parte, Mesch y otros (2002) demuestran la relevancia de ambos factores, confirmando que las personas casadas con niños conviviendo en el hogar presentan mayores tasas de voluntariado en comparación con las que se encuentran en cualquier otra situación familiar.

Finalmente, Nesbit (2012) estudia el comportamiento de los voluntarios tras algunos acontecimientos familiares importantes en sus vidas, concluyendo que el nacimiento de un 
hijo disminuye la actividad del voluntariado, que los hombres divorciados, así como los divorciados con hijos en general, incrementan su actividad como voluntarios. Asimismo, esta dedicación disminuye tras enviudar, aunque entre las personas viudas de mayor edad la ratio se invierte.

\section{F. Trabajo remunerado frente a trabajo voluntario}

Grant (2012) sugiere que las profesiones también juegan un papel crítico en la configuración de los motivos que inducen a los individuos a realizar actividades de voluntariado. El desempeño y la demanda de diferentes roles sociales (Rapaport y Rapaport, 1978; Gross, 1980) establecen una relación negativa entre las horas de trabajo remunerado y las dedicadas a voluntariado, tal y como confirma Vaillancourt (1994) en el caso de la realidad social Canadiense. Desde ese punto de vista, los trabajadores a tiempo parcial se involucrarían más en actividades de voluntariado que los trabajadores a tiempo completo.

Además, la investigación realizada por Gómez y Guntherson (2003) sobre voluntariado y condiciones laborales, demuestra que la probabilidad de trabajo voluntario se reduce en el trabajo por turnos, e incrementa en el trabajo a tiempo parcial, bajo presión, y cuando se realiza trabajo en el domicilio particular. Sin embargo, ni el trabajo nocturno, ni la flexibilidad de horarios, ni la concentración del trabajo semanal, ni la sobre-cualificación, conducen a una mayor o menor actividad de voluntariado.

\section{G. Trabajo voluntario y tamaño de la población}

No sólo la actividad laboral puede ser un facilitador $-\mathrm{o}$ no- de la mayor o menor dedicación al voluntariado, sino que también los aspectos culturales y la propia comunidad que rodea al individuo pueden tener impacto en la actividad de voluntariado, al margen de las características personales, las condiciones organizativas y las laborales. Algunos autores encuentran una relación inversa entre el tamaño de la localidad y la propensión al voluntariado. Así, Vaillancourt (1994) afirma que el voluntariado es más frecuente entre personas que viven en municipios pequeños. García y Marcuello (2007) demuestran este hecho, confirmando que el voluntariado es mayor en las ciudades pequeñas -entre $10.000 \mathrm{y}$ 100.000 habitantes- seguidas por las localidades que tienen entre 100.000 y 400.000 habitantes. 


\section{VALORES SECULARES Y TRADICIONALES}

Al hablar de valores culturales tradicionales y seculares, Inglehart y Baker (2000) utilizan el término "tradicional" en un sentido específico. Las sociedades tradicionales son en cierta medida más autoritarias, manifiestan una escasa tolerancia hacia cuestiones como el aborto, el divorcio, y la homosexualidad; tienden a conceder más protagonismo al papel dominante de los hombres en la economía y la política; y refuerzan el reconocimiento de la autoridad de los padres y la importancia de la familia. Finalmente, la mayoría de las tradiciones conceden a la religión un papel central en la sociedad. Frente a este panorama, las sociedades más avanzadas, de cariz secular, presentarían las características contrarias.

En la extensa literatura sobre la incidencia de los valores culturales en la actividad del voluntariado, la importancia de la religión y la orientación religiosa han sido propuestos frecuentemente como factores explicativos de la dedicación altruista a ciertas actividades (Jackson y otros, 1995; Wilson y Musick, 1997; Grønbjerg y Never, 2004; Jones, 2006; Brown y Ferris, 2007; Wang y Graddy, 2008; Choi y Dinitto, 2012; Forbes y Zampelli, 2014; Prouteau y Sardinha, 2013). En este sentido, Grønbjerg y Never (2004) demuestran que el compromiso con la religión es una de las variables explicativas más importantes del voluntariado entre los residentes de Indiana, así como que las motivaciones religiosas ejercen una incidencia positiva en las preferencias sobre dos tipos de voluntariado concretos: como era de esperar, en el que se desarrolla en instituciones religiosas, pero también en las dedicadas a la prestación de servicios directos a la comunidad, con independencia de la naturaleza de la institución que las impulse. Por el contrario, Prouteau y Sardinha (2013) afirman que a pesar de que existen estudios que encuentran cierta relación entre la religiosidad y la dedicación al voluntariado, en algunas ocasiones sus resultados son contradictorios.

$\mathrm{Al}$ poner el foco de atención en la esfera de las donaciones de fondos, tanto Brown y Ferris (2007) como Choi y Dinitto (2012) concluyen que existen variables sociodemográficas como la edad, el estado civil, el nivel educativo o la raza que ayudan a explicar las preferencias a la hora de colaborar con causas de carácter religioso o secular. Sobre este asunto, Wang y Graddy (2008) demuestran que el capital social que se genera con la mayor confianza tanto en las instituciones como en las personas, la existencia de redes sociales formales e informales y el mayor compromiso de la ciudadanía, incrementan la participación en organizaciones benéficas en general. A la vez, existen valores como el activismo social que 
sólo contribuyen a un incremento de la colaboración con causas seculares, mientras que otros como la satisfacción en la vida, estimulan las donaciones dirigidas a obras de carácter religioso.

Finalmente, aunque la influencia de la religión sobre el voluntariado ha sido ampliamente estudiada, la dicotomía entre valores seculares y tradicionales no ha sido analizada aún como factor explicativo. Sin embargo, el hecho de que algunos trabajos previos hayan utilizado esta clasificación en la predicción de otros aspectos, como por ejemplo, la satisfacción en el trabajo o en la vida (Georgellis y Lange, 2012), hace pensar que también existe alguna relación directa con la actitud y las motivaciones de las personas que deciden dedicar parte de su tiempo al voluntariado, en general, y a algunos tipos más que a otros, en particular.

\section{HIPÓTESIS}

En los apartados previos se han puesto de manifiesto dos conclusiones relevantes. Por un lado, la disparidad de resultados acerca de los factores sociodemográficos y contextuales que condicionan la dedicación al voluntariado. Por otro, la ausencia de estudios que analicen la influencia que ejercen los valores -seculares o tradicionales- sobre el perfil de los voluntarios en Europa, así como sobre sus preferencias a la hora de decidir entre distintos tipos de voluntariado, aunque la religiosidad y las motivaciones del voluntariado de tipo religioso, si hayan sido cuestiones ampliamente analizadas. Al mismo tiempo, siguiendo a Grönlund (2012) se podría plantear si otras ideologías de tipo secular, al margen de las de carácter religioso, promocionan de algún modo la actividad del voluntariado, lo que permitiría ahondar en el conocimiento de sus motivaciones.

Precisamente, en este estudio se pretende arrojar algo de luz sobre estos asuntos, planteando para ello las siguientes hipótesis de investigación:

H1: El perfil sociodemográfico y los valores culturales de los voluntarios son distintos al de las personas que no dedican tiempo al voluntariado.

El contraste de esta hipótesis permitirá establecer una caracterización del perfil del voluntariado en Europa. 
H2: Las personas que colaboran como voluntarios en organizaciones de tipo de religioso presentan un perfil en el que predominan los valores tradicionales frente a los seculares.

H3: Las personas que colaboran como voluntarios en organizaciones sindicales presentan un perfil en el que predominan los valores seculares frente a los tradicionales.

El contraste de estas dos últimas hipótesis demostrará si existe alguna correspondencia entre los valores culturales y la entrega a un determinado tipo de voluntariado, ya sea este de carácter religioso o secular.

\section{ESTUDIO EMPÍRICO}

\section{A. Metodología}

La metodología utilizada para el cumplimiento de los objetivos de este trabajo se fundamenta en el modelo de regresión logística binaria, un caso particular de los denominados modelos de regresión con respuesta dicotómica. Esta técnica estadística permite determinar la probabilidad de ocurrencia del suceso investigado -en el caso que nos ocupa, dedicar o no dedicar tiempo a actividades de voluntariado $\mathrm{y}$, entre los que lo hacen, inclinarse hacia el voluntariado de tipo religioso o secular- frente a la probabilidad de ocurrencia del suceso contrario.

\section{B. Selección de la muestra}

Los datos empleados en la presente investigación se han obtenido de la última European Values Study (EVS-2008), realizada por la EVS Foundation. Se trata de una encuesta longitudinal de investigación sobre los valores humanos básicos realizada los años 1981 (16 países), 1990 (29 países), 1999/2000 (33 países) y 2008 (46 países). A través de ella se obtiene información detallada sobre las ideas, creencias, preferencias, actitudes, valores y opiniones de los ciudadanos de toda Europa: 1) percepción de la vida, 2) trabajo, 3 ) religión, 4) familia y matrimonio, 5) política y sociedad, 6) actitudes morales, 7) identidad nacional y 8) medioambiente. La población objeto de estudio son personas de mayores de 18 años. El trabajo de campo permitió la obtención de más de 56.000 encuestas válidas. 
Dados los objetivos de la presente investigación, se obtuvo una submuestra aleatoria de 27.008 personas, de las cuales el 49,6\% dedicaban tiempo a tareas de voluntariado de diferente índole (político, religioso, sindical, ecologista...), mientras que el resto confesaba que nunca participaba en este tipo de actividades. En conjunto, el 46,2\% de los integrantes de la muestra eran hombres y el 53,8\% mujeres. Este gap de género se intensifica entre los no voluntarios (43,0\% de hombres frente al $57,0 \%$ de mujeres) y se reduce entre los voluntarios, hasta casi igualarse ambos colectivos (49,5\% de hombres y 50,5\% de mujeres). La edad media de los encuestados era de 46,5 años, sin que apenas existan diferencias entre voluntarios (46,3 años) y no voluntarios (46,7 años). El 26,6\% de los encuestados tiene un nivel de formación bajo (31,9\% en no voluntarios y 21,3\% entre los que realizan actividades de voluntariado), el $45,9 \%$ posee estudios de nivel medio (47,2\% frente al 44,6\%, respectivamente) y el $27,5 \%$ de nivel alto. Los estudios superiores son menos comunes entre las personas que no hacen voluntariado $(20,9 \%)$ y más frecuentes entre los que sí lo practican $(34,1 \%)$.

Otro aspecto a tener en consideración es que el voluntariado parece una actividad más propia de personas con niveles de ingresos elevados: el 49,6\% de los voluntarios declaran este nivel de ingresos, un porcentaje que desciende hasta el $28,6 \%$ entre los no voluntarios.

Finalmente, y aunque en principio se pudiera pensar lo contrario, las restricciones temporales derivadas de la situación profesional no constituyen un impedimento para la dedicación al voluntariado entre los individuos de la muestra. De este modo, más de la mitad de los voluntarios, concretamente el $51,7 \%$, son autoempleados a tiempo completo o asalariados con una dedicación superior a las 30 horas semanales, mientras que esta ratio desciende al 43,1\% entre los no voluntarios. Entre los individuos con más tiempo libre, inactivos o asalariados a jornada parcial, predominan los que no se dedican a las actividades de voluntariado: el 57,0\% de los no voluntarios se encuentran en estas categorías, frente al $48,2 \%$ de los que sí realizan tareas de voluntariado.

\section{Definición de variables}

\section{C.1. Variable dependiente}

La variable dependiente de este estudio es la actividad de voluntariado. A los encuestados se les preguntó acerca de si trabajaban voluntariamente y de manera no retribuida para una serie de organizaciones de diversa índole: organizaciones de bienestar social, 
religiosas, actividades culturales, sindicales, partidos o grupos políticos, comunidades de acción local, defensa de los derechos humanos, medioambiente, ecologistas o derechos de los animales. De manera general, cualquier individuo que dedicara algo de su tiempo a alguna de estas actividades ha sido clasificado como voluntario, con independencia de la cantidad de tiempo empleado. Los encuestados en esta situación fueron codificados como 1, mientras que los individuos que no participaban en ninguna de estas actividades de voluntariado se codificaron como 0.

\section{C.2. Variables independientes}

Teniendo en consideración los estudios previos sobre la actividad de voluntariado, y para poder contrastar las hipótesis de investigación planteadas en el apartado previo, las variables que integrarán el modelo de regresión se agrupan en dos categorías: factores de naturaleza sociodemográfica por un lado, y valores culturales por otro. Estos últimos se clasifican a su vez en dos tipos: seculares y tradicionales. Las características inherentes a los valores implícitos en cada uno de ellos han sido resumidas por Inglehart y Baker (2000) en diez variables clave que pueden observarse en la Tabla 1, en la que se incluyen la relación y codificación de todas las variables explicativas consideradas en la investigación. 
Tabla 1: Variables explicativas: codificación

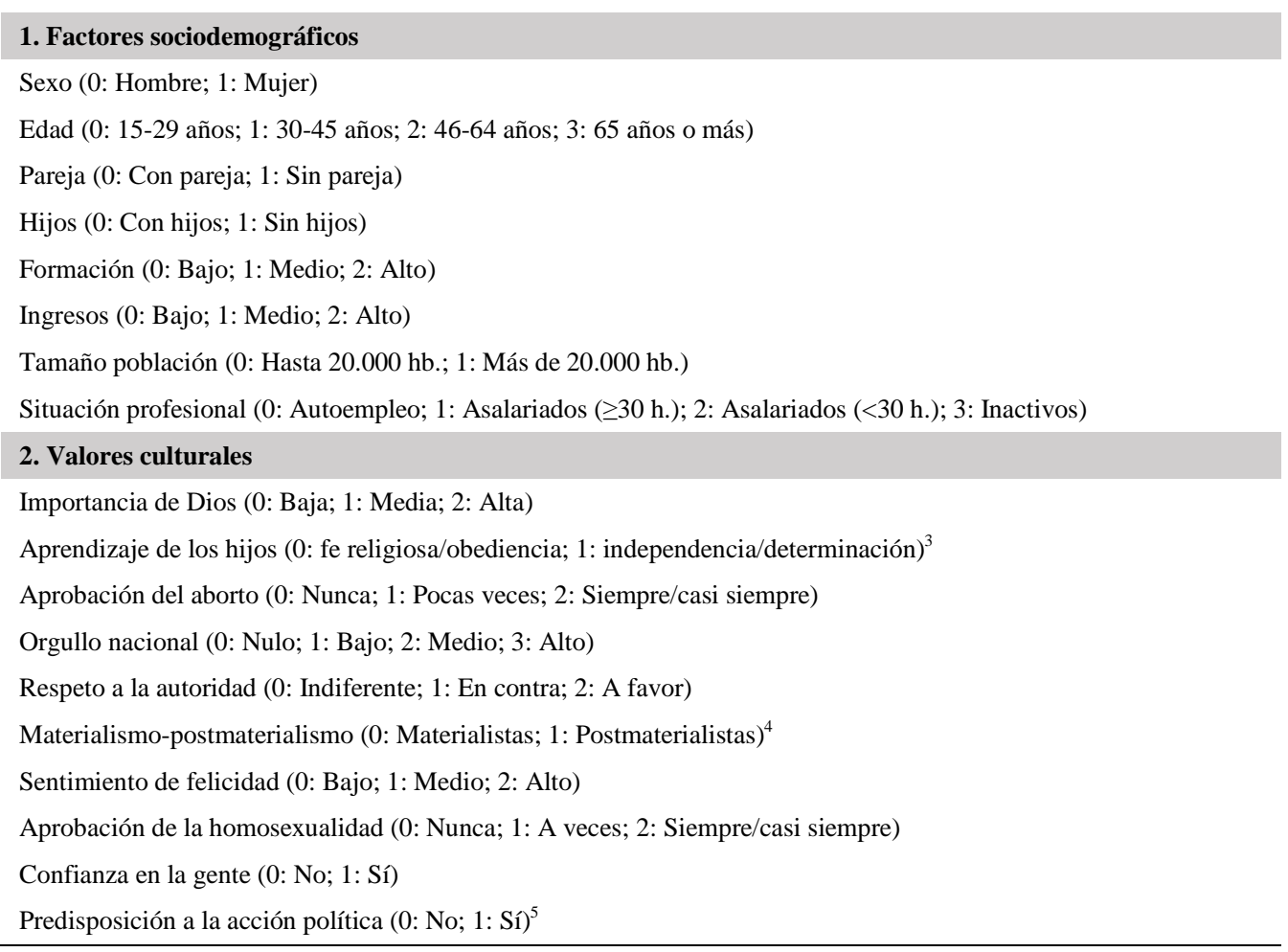

Fuente: Elaboración propia

\section{ANÁLISIS DE RESULTADOS}

\section{A. Análisis bivariante}

El objetivo general de este trabajo trata de identificar el perfil de los individuos que participan en las actividades de voluntariado en Europa, así como los factores que influyen en sus preferencias a la hora de optar por un tipo de trabajo voluntario u otro. Con ello se pretende averiguar si ciertas características sociodemográficas, unido a los valores seculares o tradicionales del individuo, explican de algún modo, tanto el nivel de participación en actividades de voluntariado, como la elección que realizan los voluntarios. Para lograr este fin se utiliza en primer lugar un análisis de tablas de contingencia y Chi-cuadrado de Pearson, con el objeto de examinar la posible relación bivariante entre la variable dependiente -estar implicado o no en actividades de voluntariado- y un conjunto de variables independientes

\footnotetext{
${ }^{3}$ A los encuestados se les preguntó acerca de las cualidades que se deberían enseñar e inculcar a los hijos: fe y obediencia o, por el contrario, independencia y determinación.

${ }^{4}$ Se presentó al encuestado una lista para que eligiera de entre los siguientes objetivos los dos más importantes: 1. "mantener el orden en la nación", 2. "dar a la gente más voz en las decisiones importantes del gobierno", 3. "lucha contra el aumento de los precios" y 4. "proteger la libertad de expresión". Los individuos con valores materialistas son los que eligieron las opciones 1 y 3 de la lista anterior, mientras que los sujetos con valores postmaterialistas se decantaron por las opciones 2 y 4 .

5 En este caso se solicitó al encuestado que manifestara su predisposición a firmar peticiones de adhesión a diferentes causas, y bajo qué circunstancias estaría dispuesto a ello.
} 
agrupadas en las dos categorías expuestas anteriormente. Esta aproximación supone una preparación para el análisis multivariante que se realizará posteriormente, ya que en el modelo de regresión logística sólo se deberían incluir aquellas variables independientes que tienen una capacidad de predicción estadísticamente significativa.

De este modo, la aplicación del contraste de Pearson a un nivel de significación de 0,05 nos lleva a identificar la falta de capacidad explicativa en el modelo de dos de las variables consideradas inicialmente. Estas variables son, por un lado, la variable sociodemográfica que hace referencia al sexo de los encuestados ${ }^{6} \mathrm{y}$, por otro, la variable relativa al grado de aceptación de la homosexualidad entre los individuos de la muestra. Por esta razón, ambas variables han sido excluidas del análisis multivariante.

\section{B. Análisis multivariante}

Tras el análisis exploratorio inicial, a continuación se plantea un modelo de regresión logística, haciendo uso de todas las variables predictoras que han resultado ser significativas, para evaluar, en una primera fase del estudio, cuál es la combinación de variables que mejor explica la probabilidad de que un individuo dedique su tiempo al trabajo como voluntario.

En el Modelo 1 de la Tabla 2 se presentan las variables que actuando conjuntamente contribuyen mejor a configurar el perfil de las personas que realizan actividades de voluntariado en Europa, en comparación con las que no desempeñan este tipo de trabajo. De este modo, a nivel sociodemográfico se puede afirmar que la propensión al voluntariado aumenta con la edad, con el nivel de formación y con los ingresos, siendo más frecuente en localidades menos pobladas. En el ámbito de los valores culturales, el voluntariado es más probable entre las personas que sienten un elevado orgullo nacional, que respetan la autoridad, imbuidos de valores postmaterialistas, que se sienten felices, confían en la gente y se encuentran predispuestos a la acción política. Todos los resultados presentados son significativos en el nivel del $1 \%$.

Para este nivel de significación, el modelo de regresión logística indica que el resto de variables no se relacionan -ni positiva, ni negativamente- con la propensión a dedicar tiempo personal a labores de voluntariado, en general. Según los resultados obtenidos, ni el hecho de

\footnotetext{
${ }^{6}$ Este resultado es coherente con los estudios que sugieren que en Europa no existen diferencias por razón de género en cuanto a la dedicación al voluntariado (Hodgkinson y Weitzman, 1996; Gaskin y Smith, 1997; Hall y otros, 1998).
}

REVESCO No 120 - Primer Cuatrimestre 2016 - ISSN: 1885-8031 - www.ucm.es/info/revesco 
vivir o no en pareja, ni el tener hijos, ni la situación profesional afectarían a la decisión de trabajar como voluntario. Entre los valores culturales, ni la importancia de Dios, ni el aprendizaje de los hijos, ni la aprobación o desaprobación del aborto, ejercen ninguna influencia en el perfil del voluntariado.

A pesar de todo, el impacto de cada una de las variables significativas en la probabilidad de dedicar tiempo al voluntariado difiere sustancialmente de unas a otras, tal y como indica el análisis de los intervalos de confianza obtenidos para las correspondientes odds ratios (ver Tabla 2). Las variables que contribuyen de manera más decisiva a predecir las actividades de voluntariado serían, por este orden, las siguientes: la predisposición del voluntario a la acción política (OR: 1,7; IC: 1,461-1,978), los valores postmaterialistas (OR: 1,696; IC: 1,437-2,000), los ingresos personales (OR: 1,549; IC: 1,406-1,706) y la confianza en el prójimo (OR: 1,379; IC: 1,184-1,607). En cuanto al tamaño de la localidad de residencia (OR: 0,663; IC: 0,574-0,766), la propensión el voluntariado es mayor cuanto menor es el tamaño poblacional. 
Tabla 2: Regresión logística: factores que determinan la actividad de voluntariado

\begin{tabular}{|c|c|c|c|}
\hline \multirow{3}{*}{ Variables } & \multicolumn{3}{|c|}{ Odds ratios $95 \%$ I.C. de OR } \\
\hline & \multicolumn{3}{|c|}{ Modelo1 General } \\
\hline & OR & Inferior & Superior \\
\hline \multicolumn{4}{|l|}{ 1. Factores sociodemográficos } \\
\hline Edad & 1.077 & 1.000 & 1.159 \\
\hline Pareja & & & \\
\hline Hijos & & & \\
\hline Formación & 1.253 & 1.127 & 1.392 \\
\hline Ingresos & 1.549 & 1.406 & 1.706 \\
\hline Tamaño población & 0.663 & 0.574 & 0.766 \\
\hline \multicolumn{4}{|l|}{ Situación profesional } \\
\hline \multicolumn{4}{|l|}{ 2. Valores } \\
\hline \multicolumn{4}{|l|}{ Importancia de Dios } \\
\hline \multicolumn{4}{|l|}{ Aprendizaje de los hijos } \\
\hline \multicolumn{4}{|l|}{ Aprobación del aborto } \\
\hline Orgullo nacional & 1.144 & 1.038 & 1.260 \\
\hline Respeto a la autoridad & 1.091 & 1.002 & 1.189 \\
\hline Materialismo-postmaterialismo & 1.696 & 1.437 & 2.000 \\
\hline Sentimiento de felicidad & 1.154 & 1.022 & 1.304 \\
\hline Confianza en la gente & 1.379 & 1.184 & 1.607 \\
\hline Predisposición a la acción política & 1.700 & 1.461 & 1.978 \\
\hline Constante & 0.174 & & \\
\hline$\chi^{2}$ Test de eficiencia- Modelo global & 483.96 & & \\
\hline Grados de libertad & 10 & & \\
\hline Nivel de significación & 0.000 & & \\
\hline \multicolumn{4}{|l|}{ \% Predicción correcto: } \\
\hline Global & $65.6 \%$ & & \\
\hline Voluntarios & $69.1 \%$ & & \\
\hline No voluntarios & $61.8 \%$ & & \\
\hline
\end{tabular}

Fuente: Elaboración propia

El estadístico de contraste aplicado para evaluar la validez del modelo en su conjunto indica que existen razones suficientes para aceptar la validez del mismo ${ }^{7}$, es decir, para afirmar que el hecho de que un individuo dedique o no tiempo personal a actividades de voluntariado puede ser satisfactoriamente explicado por el conjunto de variables sociodemográficas y del ámbito de los valores culturales- consideradas en la presente investigación. Estos resultados confirmarían el planteamiento realizado en la primera de las hipótesis de investigación, que consideraba que el perfil del voluntario europeo está

\footnotetext{
${ }^{7}$ La prueba ómnibus del modelo, utilizada para este fin, presenta los siguientes resultados: Chi Cuadrado: 483,96; Sig. 0,0000.
} 
determinado por factores sociodemográficos y por el catálogo de valores culturales, contribuyendo todo ello a la caracterización del voluntariado en Europa.

En todo caso, la capacidad de generalización del modelo, aunque aceptable, resulta limitada, ya que sólo el $65,6 \%$ de los individuos considerados resultó bien clasificado conociendo de antemano su situación real. Existen pequeñas diferencias entre los porcentajes correspondientes a voluntarios $(69,1 \%)$ y no voluntarios $(61,8 \%)$, lo que apunta a que el perfil de los primeros resulta más fácil de predecir. Estos resultados sugieren que existen otros factores al margen de los presentados en este primer modelo que contribuyen a comprender las razones de la mayor implicación de los individuos en las actividades de voluntariado.

Esta circunstancia puede venir explicada por la heterogeneidad en cuanto a los tipos de voluntariado incluidos en la EVS, lo que probablemente enmascare realidades muy diversas. Por ejemplo, el perfil personal de un individuo que trabaja de manera voluntaria en una asociación profesional puede ser muy diferente del que se vuelca en la defensa de los derechos humanos, se entrega a la causa pacifista o dedica su tiempo de forma desinteresada a la salvaguarda del medio ambiente. Asimismo, los valores individuales de estos sujetos pueden presentar diferencias significativas que hayan quedado diluidas en el modelo general obtenido en un primer momento.

Esta realidad justifica la estimación de dos nuevos modelos de regresión que incluyen a colectivos más homogéneos intragrupo $\mathrm{y}$, a priori, más heterogéneos intergrupos. Concretamente, de la muestra general se extrajeron dos submuestras de personas que se dedicaban al voluntariado en organizaciones religiosas, frente a individuos que dedican tiempo no retribuido al voluntariado en organizaciones sindicales. El objetivo es determinar los perfiles individuales de ambos colectivos para analizar las posibles similitudes o diferencias que existan entre ellos. Los resultados de los dos modelos de regresión logística se resumen en la Tabla 3.

Al comparar uno y otro se aprecia que existen un total de seis factores coincidentes que son significativos a la hora de explicar ambos modelos, y que conforman el tronco común de la mayor o menor propensión hacia cualquiera de los dos tipos de voluntariado analizados: tres variables sociodemográficas (edad, nivel de ingresos y tamaño de la población) y tres valores culturales (importancia de Dios, aprobación del aborto y confianza en la gente). La 
influencia de estas seis variables ha de interpretarse en todos los casos en sentido opuesto en uno y otro tipo de voluntariado.

De este modo, a nivel sociodemográfico hay que destacar que los que se dedican al voluntariado en organizaciones sindicales son más jóvenes, obtienen menos ingresos y habitan en poblaciones más grandes, con más de 20.000 habitantes; justo lo contrario de lo que define a las personas que dedican tiempo no retribuido a organizaciones de ámbito religioso.

Trasladándonos al terreno de los valores, los voluntarios sindicales conceden poca importancia a Dios, aprueban el aborto y confían menos en los demás. Los resultados obtenidos en cuanto al voluntariado religioso demuestran todo lo contrario, destacando la importancia de Dios como el valor más relevante de todos.

Respecto a los factores explicativos que son propios y exclusivos de cada uno de los tipos de voluntariado, en el caso del sindical irrumpen el nivel de formación y la situación laboral. Estos voluntarios se caracterizan por su mayor nivel educativo, y por una situación laboral donde destaca una mayor dedicación de horas a la jornada de trabajo. Sin embargo, ninguno de estos dos aspectos incide en el perfil del voluntariado de tipo religioso. Por su parte, hay dos valores que sólo están presentes en el perfil de estos últimos: los valores postmaterialistas y la mayor predisposición a la acción política.

Finalmente y actuando de forma conjunta, hay cuatro variables que no son representativas de las preferencias de los voluntarios en ninguno de los dos modelos analizados: el hecho de convivir en pareja o de tener hijos, el orgullo nacional y el respeto a la autoridad.

Los resultados obtenidos demuestran que los valores culturales, seculares o tradicionales, inciden en los tipos de voluntariado sindical o religioso, confirmando de esta forma las hipótesis $\mathrm{H} 2$ y $\mathrm{H} 3$ del estudio. Los modelos clasifican correctamente a un 71,5\% de los voluntarios de tipo religioso en el Modelo 2 y a un 76,7\% de las personas que realizan una actividad de voluntariado sindical en el Modelo 3, lo que confirma la mejora del carácter predictivo de las variables investigadas cuando se alinean con un tipo de voluntariado concreto. 
Tabla 3: Regresión logística: factores que determinan la actividad de voluntariado religioso y sindical

\begin{tabular}{|c|c|c|c|c|c|c|}
\hline \multirow{3}{*}{ Variables } & \multicolumn{6}{|c|}{ Odds ratios 95\% I.C. de OR } \\
\hline & \multicolumn{3}{|c|}{ Modelo 2 Religioso } & \multicolumn{3}{|c|}{ Modelo 3 Sindical } \\
\hline & OR & Inferior & Superior & OR & Inferior & Superior \\
\hline \multicolumn{7}{|l|}{ 1. Factores sociodemográficos } \\
\hline $\begin{array}{l}\text { Edad } \\
\text { Pareja } \\
\text { Hijos }\end{array}$ & 1.252 & 1.060 & 1.480 & 0.725 & 0.564 & 0.932 \\
\hline Formación & & & & 1.393 & 0.995 & 1.951 \\
\hline Ingresos & 1.541 & 1.227 & 1.936 & 0.576 & 0.418 & 0.792 \\
\hline Tamaño población & 0.467 & 0.329 & 0.663 & 2.142 & 1.323 & 3.470 \\
\hline Situación profesional & & & & 0.786 & 0.618 & 0.999 \\
\hline \multicolumn{7}{|l|}{ 2. Valores } \\
\hline $\begin{array}{l}\text { Importancia de Dios } \\
\text { Aprendizaje de los hijos }\end{array}$ & 3.248 & 2.481 & 4.252 & 0.261 & 0.181 & 0.375 \\
\hline $\begin{array}{l}\text { Aprobación del aborto } \\
\text { Orgullo nacional } \\
\text { Respeto a la autoridad }\end{array}$ & 0.718 & 0.566 & 0.910 & 1.430 & 1.049 & 1.951 \\
\hline $\begin{array}{l}\text { Materialismo-postmaterialismo } \\
\text { Sentimiento de felicidad }\end{array}$ & 1.540 & 1.003 & 2.366 & & & \\
\hline Confianza en la gente & 1.970 & 1.354 & 2.867 & 0.585 & 0.357 & 0.958 \\
\hline Predisposición a la acción política & 2.431 & 1.659 & 3.563 & & & \\
\hline Constante & 0.070 & & & 17.336 & & \\
\hline $\begin{array}{l}\chi^{2} \text { Test de eficiencia- Modelo global } \\
\text { Grados de libertad } \\
\text { Nivel de significación } \\
\end{array}$ & $\begin{array}{c}191.21 \\
8 \\
0.000 \\
\end{array}$ & & & $\begin{array}{c}157.21 \\
8 \\
0.000 \\
\end{array}$ & & \\
\hline \% Predicción correcto: & $71.0 \%$ & & & $74.3 \%$ & & \\
\hline Global & $71.0 \%$ & & & $74.3 \%$ & & \\
\hline Voluntariado religioso/sindical & $71.5 \%$ & & & $76.7 \%$ & & \\
\hline Otro tipo de Voluntariado & $70.5 \%$ & & & $71.4 \%$ & & \\
\hline
\end{tabular}

Fuente: Elaboración propia

\section{DISCUSIÓN Y CONCLUSIONES}

El análisis de las reacciones afectivas de los individuos frente a los contextos organizativos en los que se encuentran inmersos constituye una línea de investigación coherente con la actual tendencia del management, que sugiere la necesidad de implementar políticas de gestión basadas en las emociones, los sentimientos y los valores (Fernández y Peñalver, 2005; Fernández, 2004). En el ámbito del sector no lucrativo resultan especialmente críticos los estudios que permitan caracterizar el perfil de los voluntarios y que ayuden a comprender las razones que les inducen a entregar a los demás parte de su tiempo personal, parte de su vida. Entre esas motivaciones, que sin duda son complejas, con muchos matices y 
aristas, la literatura enfatiza la influencia de los valores y de las creencias en el voluntariado (Wilson, 2000).

Las variables sociodemográficas que configuran el perfil del voluntario han sido frecuentemente analizadas de manera aislada, aun cuando resulta evidente que nos encontramos ante un fenómeno multidimensional. La evidencia empírica se reduce de manera notable en relación con la incidencia de los valores culturales en la caracterización del fenómeno, mientras que no existen estudios que analicen el efecto conjunto de ambos grupos de variables, lo que aporta valor y significado al presente trabajo.

De este modo, los primeros resultados obtenidos demuestran que dedicarse al voluntariado, en general, depende de una combinación de variables agrupadas en dos categorías -perfil sociodemográfico y valores culturales-. La demostración de esta dependencia confirma el planteamiento realizado en la primera de las hipótesis de investigación relativo a la multidimensionalidad del modelo. Las variables que, actuando conjuntamente, contribuyen de manera más decisiva a predecir el perfil de las personas que realizan actividades de voluntariado son: la predisposición a la acción política, la importancia que conceden a los valores postmaterialistas, el nivel de ingresos, la confianza en el prójimo y el tamaño de la población en la que residen.

Sorprende que el hecho religioso no aparezca en el modelo de regresión logística como un factor significativo dentro de la combinación que mejor explica el perfil del voluntario, a pesar de que tradicionalmente la religiosidad se ha venido utilizando para justificar esta realidad (por ejemplo Grönlund, 2012). Este resultado puede interpretarse en el sentido de que el voluntariado depende más del compromiso personal que de su confesionalidad religiosa. En esta línea, cabría preguntarse si la identificación de determinadas causas con la confesionalidad de la institución que las promueve suma voluntades o, por el contrario, resta apoyos por la asociación que algunos individuos puedan realizar entre la misión y el carácter religioso de la organización que la respalda. Esta reflexión podría servir a los gestores de las entidades no lucrativas a la hora de decidir dónde poner el énfasis en las campañas para la captación de voluntarios: bien en el carácter de los proyectos o bien en la naturaleza de la institución.

Sin embargo, todas estas consideraciones previas deben interpretarse con cautela ya que la capacidad explicativa del modelo obtenido, aunque suficiente, es limitada $(65,6 \%)$. 
Este hecho pude venir motivado porque los valores culturales que conforman la identidad del sujeto no inciden en el voluntariado "a ciegas", sino que es muy probable que los valores individuales orienten esa elección hacia diferentes tipos de voluntariado.

Todo lo expuesto hasta el momento sigue la línea de aquellos trabajos previos que han abordado la cuestión del voluntariado como un fenómeno único, indiferenciado. Sin embargo, conscientes de que no se trata de una realidad que se pueda abordar fácilmente de manera global, en esta investigación se ha analizado el perfil de los voluntarios según la finalidad de la actividad a la que dedican su tiempo de manera altruista, ya que un estudio global podría estar enmascarando realidades muy diversas y heterogéneas.

Por este motivo, se han elegido dos tipos de voluntariado a priori distantes en cuanto a su orientación: el voluntariado de trasfondo religioso y el voluntariado de corte sindical, aun siendo conscientes de su diferente grado de representatividad. En estos dos tipos de voluntariado la incidencia de los valores seculares y tradicionales parece bastante evidente. Este nuevo planteamiento mejora la eficacia clasificatoria global de ambos modelos $(71,0 \%$ en el caso del voluntariado religioso y $74,3 \%$ en el sindical), demostrando que los valores culturales condicionan la elección de los individuos. Este hecho conecta con los planteamientos realizados en las hipótesis segunda y tercera. En concreto, el análisis de los dos modelos de regresión logística pone de manifiesto que los factores que explican el voluntariado religioso son distintos a los que determinan el de carácter sindical. En unos casos porque las variables explicativas son diferentes, y en otros porque la influencia de las variables actúa en sentido contrario.

Por una parte, el voluntariado religioso viene condicionado por dos variables presentes específicamente en este grupo: la mayor predisposición a la acción política y los valores de naturaleza postmaterialista entre sus miembros, lo que podría ser reflejo del compromiso social que se presupone en este tipo de individuos. En contraste, las variables idiosincráticas del modelo sindical son una mayor formación y la situación profesional, lo que responde al carácter más utilitarista de este tipo de voluntariado.

Por otra parte, existe un grupo de variables sociodemográficas y de valores culturales que, formando parte integrante de ambos modelos, marcan aún más la diferencia entre ambos ya que influyen en sentido opuesto. Así, el perfil del voluntariado religioso se manifiesta entre las personas de más edad, con mayores ingresos y que viven en poblaciones más pequeñas, 
mientras que los voluntarios sindicales son más jóvenes, obtienen menores ingresos y residen en poblaciones más grandes. Desde el punto de vista de los valores que caracterizan a un grupo y otro, los voluntarios religiosos conceden una gran importancia a Dios y desaprueban el aborto a la vez que confían más en la gente, justo lo contrario de lo que ocurre entre las personas que realizan un voluntariado de tipo sindical. La desconfianza en la gente, entre los que donan tiempo de forma desinteresada a organizaciones sindicales, contrasta con la actitud altruista inherente a las actividades de voluntariado.

Siguiendo la clasificación de valores seculares y tradicionales de Inglehart y Welzel (2005), se puede afirmar que entre los voluntarios religiosos predominan los valores de naturaleza tradicional, confirmándose de este modo el enunciado de la segunda hipótesis del trabajo. Al mismo tiempo, la mayor presencia de los valores seculares entre los voluntarios sindicales corrobora los planteamientos de la hipótesis tercera.

Para finalizar nos gustaría poner de manifiesto que con esta investigación no se pretende agotar el tema de la influencia de los valores sobre el voluntariado, sino que se abre la puerta para que en futuros trabajos abordemos la cuestión desde una doble vertiente. Por un lado, ampliando el análisis hacia otros tipos de voluntariado y de las organizaciones que los promueven. Por otro lado, enfrentando la Teoría de los valores básicos propuesta por Schwartz (1992), con la que defienden Inglehart y Wenzel (2005) en cuanto a su incidencia sobre el comportamiento del voluntariado.

\section{BIBLIOGRAFÍA}

ANSHELL, C. y GASH, A. (2008) Collaborative Governance in Theory and Practice, Journal of Public Administration Research and Theory, vol. 18, $\mathrm{n}^{\circ}$ 4, pp. 543-571.

BRADSHAW, Y. W. y WALLACE, M. (1996) Global inequalities, Sage.

BRADY, H., SCHLOZMAN, K. L. y VERBA, S. (1999) Prospecting for participants: rational expectations and the recruitment of political activists, American Political Science Review, $\mathrm{n}^{\circ} 93$, pp. 153-169.

BRADY, H., VERBA, S. y SCHLOZMAN, K.L. (1995) Beyond SES: a resource model of political participation, American Political Science Review, nº 89, pp. 269-295.

BREHM, J. y RAHN, W. (1997) Individual-Level Evidence for the Causes and Consequences of Social Capital, American Journal of Political Science, 41, 3 (July), pp. 999-1023. 
BROWN, E. y FERRIS, J. M. (2007) Social capital and philanthropy: An analysis of the impact of social capital on individual giving and volunteering, Nonprofit and Voluntary Sector Quarterly, vol. 36, n 1, pp. 85-99.

CAPPELLARI, L. y TURATI, G. (2004) Volunteer Labour Supply: The Role of Workers' Motivations, Annals of Public and Cooperative Economics, vol. 75, $\mathrm{n}^{\circ} 4$.

CHOI, N. G. y DINITTO, D. M. (2012) Predictors of Time Volunteering, Religious Giving, and Secular Giving: Implications for Nonprofit Organizations, Journal of Sociology \& Social Welfare, vol. 39, nº 2, pp. 93-120.

DAY, K. M. y DEVLIN, R. A. (1996) Volunteerism and crowding out: Canadian econometric evidence, Canadian Journal of Economics, vol. 29, n 1, pp. 37-53.

DIMAGGIO, P. (1994) Culture and economy, in N. Smelster and R. Swedberg (eds), The Handbook of Economic Sociology, Princeton: Princeton University Press.

EINOLF, C. J. (2011) Gender differences in the correlates of volunteering and charitable giving, Nonprofit and Voluntary Sector Quarterly, vol. 40, nº 6, pp. 1092-1112.

FERNÁNDEZ, A. J. (2004) Feelings Management. La Gestión de los sentimientos organizativos, Mind-Value. La Caixa.

FERNÁNDEZ, A. J. y PEÑALVER, O. (2005) Management, una aplicación práctica: la gestión de los sentimientos organizativos. Druk Comunicación.

FORBES, K. F. y ZAMPELLI, E. M. (2014) Volunteerism: The Influences of Social, Religious, and Human Capital, Nonprofit and Voluntary Sector Quarterly, vol. 43, $\mathrm{n}^{\mathrm{o}} 2$, pp. 227-253.

FREEMAN, R. (1997) Working for nothing: the supply of volunteer labor, Journal of Labor Economics, n ${ }^{\circ} 15$, pp. 140-167.

GALLAGHER, S. (1994) Doing their share: comparing patterns of help given by older and younger adults, Journal of Marriage and Family, no 56, pp. 567-578.

GARCÍA, I. y MARCUELLO, C. (2007) Social capital and participation in Nonprofit Organizations, Nonprofit and Voluntary Sector Quarterly, vol. 36, nº 1, pp. 100-120.

GASKIN, K. y SMITH, J. (1997) A New Civic Europe? A Study of the Extent and Role of Volunteering, London, Natl. Cent. For Volun.

GEORGELLIS, Y. y LANGE, T. (2012) Traditional versus Secular Values and the Job-Life Satisfaction Relationship Across Europe, British Journal of Management, nº 23, pp. 437 454. 
GESTHUIZEN, M. y SCHEEPERS, P. (2012) Educational Differences in Volunteering in Cross-National Perspective Individual and Contextual Explanations, Nonprofit and Voluntary Sector Quarterly, vol. 41, nº 1, pp. 58-81.

GOMEZ, R. y GUNDERSON, M. (2003) Volunteer Activity and the Demands of Work and Family, Industrial Relations, vol. 58, no 4, pp. 573-589.

GRANT, A. M. (2012) Giving time, time after time: Work design and sustained employee participation in corporate volunteering, Academy of Management Review, vol. 37, $\mathrm{n}^{\circ}$ 4, pp. 589-615.

GRØNBJERG, K. A. y NEVER, B. (2004) The role of religious networks and other factors in types of volunteer work, Nonprofit Management and Leadership, vol. 14, n 3, pp. 263289.

GRÖNLUND, H. (2012) Religiousness and Volunteering: searching for connections in late modernity, Nordic Journal of Religion \& Society, vol. 25, $\mathrm{n}^{\mathrm{o}}$ 1, pp. 47-66.

GRÖNLUND, H., HOLMES, K., KANG, C., CNAAN, R. A., HANDY, F., BRUDNEY, J. L. y ZRINŠČAK, S. (2011) Cultural values and volunteering: A cross-cultural comparison of students' motivation to volunteer in 13 countries, Journal of Academic Ethics, n ${ }^{\circ} 2$, pp. 87-106.

GROSS, H. (1980) Dual career couples who live appart: two types, Journal of Marriage and the Family, no 42 , pp. 567-576.

GUTERBOCK, T. M. y FRIES, J. C. (1997) Maintaining Americal's Social Fabric: The AARP Survey of Civic Involvement. Washington D.C.: American Association of Retired Persons.

HALL, M., KNIGHTON, T., REED, P., BUSSIERE, P., MACRAE, D. y BOWEN, P. (1998) Caring Canadians, Involved Canadians, Ottowa, Statistics Canada.

HALL, M., MCKEOWN, L. y ROBERTS, K. (2001) Caring Canadians, Involved Canadians: Highlights from the 2000 National Survey of Giving, Volunteering and Participating. Catalogue no. 71-542-XIE, Ottawa: Statistics Canada.

HERZOG, A. y MORGAN, J. (1993) Formal volunteer work among older Americans, In S. Bass, F. Caro, Y. Chen (Eds.), Achieving a Productive Aging Society, 119-142, Westport CT, Auburn House.

HERZOG, A. KAHN, R. and MORGAN, J. (1989) Age differences in productive activity, The Journals of Gerontology, n 4, pp. 129-138. 
HODGKINSON, V. y WEITZMAN, M. with ABRAHAMS, J., CRUTCHFIELD, E. y STEVENSON, D. (1996) The nonprofit almanac 1996-1997: Dimensions of the independent sector, San Francisco: Jossey-Bass.

HOFSTEDE, G. H. (2001) Culture's consequences: Comparing values, behaviors, institutions and organizations across nations. Sage.

HOUSE, J. S. (1981) Social Structure and Personality, In M. Rosenberg y R.H. Turner, Social Psychology, Basic Books.

INDEPENDENT SECTOR (1999) Giving and Volunteering in the United States: Executive summary from a national survey, Internet:

www.independentsector.org/GandV/default.htm (accessed March 25, 2003).

INGLEHART, R. (1997) Modernization and postmodernization: Cultural, economic, and political change in 43 societies (vol. 19). Princeton, NJ: Princeton University Press.

INGLEHART, R. (Ed.). (2003) Human values and social change: Findings from the values surveys (vol. 89). Brill Academic Pub.

INGLEHART, R. y BAKER, W.E. (2000) Modernization, cultural change, and the persistence of traditional values, American Sociological Review, n 65, pp. 19-51.

INGLEHART, R. y WELZEL, C. (2005) Modernization, Cultural Change, and Democracy: The Human Development Sequence. New York: Cambridge University Press.

JACKSON, E. F., BACHMEIER, M. D., WOOD, J. R. y CRAFT, E. A. (1995) Volunteering and charitable giving: Do religious and associational ties promote helping behavior?, Nonprofit and Voluntary Sector Quarterly, vol. 24, $\mathrm{n}^{\circ} 1$, pp. 59-78.

JONES, K. S. (2006) Giving and volunteering as distinct forms of civic engagement: The role of community integration and personal resources in formal helping, Nonprofit and Voluntary Sector Quarterly, vol. 35, nº 2, pp. 249-266.

MENCHIK, P. L. y WEISBROD, B. A. (1987) Volunteer labor supply, Journal of Public Economics, Elsevier, vol. 32, nº 2, pp. 159-183.

MESCH, D. J., ROONEY, P. M., CHIN, W. y STEINBERG, K. S. (2002) Race and gender differences in philanthropy: Indiana as a test case, New Directions for Philanthropic Fundraising, no 37, pp. 65-77.

MUSICK, M. A., WILSON, J. y BYNUM, W. B. (2000) Race and formal volunteering: The differential effects of class and religion, Social Forces, vol. 78, n 4, pp. 1539-1570.

NATIONAL ASSOCIATION OF SECRETARIES OF STATE (1999) New Millenium Project-Phase 1: a Nationwide Study of 15-24 Year Old Youth. Washington, DC, Nat. Assoc. Secretaries of State. 
NESBIT, R. (2012) The influence of major life cycle events on volunteering, Nonprofit and Voluntary Sector Quarterly, vol. 41, nº 6, pp. 1153-1174.

O`NEILL, M. y ROBERTS, W. (1999) Giving and Volunteering in California, ARNOVA conference, Washington, D.C.

PLAGNOL, A. C., y HUPPERT, F. A. (2010) Happy to help? Exploring the factors associated with variations in rates of volunteering across Europe, Social Indicators Research, vol. 97, n 2, pp. 157-176.

PROUTEAU, L. y SARDINHA, B. (2013) Volunteering and Country-Level Religiosity: Evidence from the European Union, VOLUNTAS: International Journal of Voluntary and Nonprofit Organizations, vol. 26, pp. 242-266.

PUTNAM, R. (2000) Bowling Alone, New York: Simon \& Schuster.

RAPAPORT, R. y RAPAPORT, R. N. (1978) Working couples, New York: Harper and Row.

RASKOFF, S. y SUNDEEN, R. (1995) Trends in volunteering: An analysis of a decade, Annual meeting of Association for Research on Non-Profit Organizations and Voluntary Associations, Cleveland, Ohio.

ROSENTHAL, S., FEIRING, C., y LEWIS, M. (1998) Political volunteering from late adolescence to young adulthood, Journal of Social Issues, vol. 54, n 3, pp. 477-493.

SCHOENBERG, S. (1980) Some trends in the community participation of women in their neighborhoods, Signs, n 5, pp. 261-268.

SEGAL, L. (1993) Four essays on the supply of volunteer labor and econometrics, Unpublished doc. Diss. Northwestern Univ., Evanston, Ill.

SMITH, D. (1994) Determinants of voluntary association participation and volunteering, Nonprofit and Voluntary Sector Quarterly, n 23, pp. 243-263.

SCHWARTZ, S. H. (1992) Universals in the content and structure of values: Theoretical advances and empirical tests in 20 countries, Advances in experimental social psychology, vol. 25, no 1 , pp. 1-65.

VAILlANCOURT, F. (1994) To volunteer or not: Canada 1987, Canadian Journal of Economics, vol. 27, nº 4, pp. 813-826.

VAN INGEN, E. y DEKKER, P. (2011) Changes in the determinants of volunteering: Participation and time investment between 1975 and 2005 in the Netherlands, Nonprofit and Voluntary Sector Quarterly, vol. 40, n 4, pp. 682-702.

VOICU, B. y VOICU, M. (2009) Volunteers and volunteering in Central and Eastern Europe, Sociológia, vol. 41, nº 6, pp. 539-563. 
WANG, L. y GRADDY, E. (2008) Social capital, volunteering, and charitable giving, Voluntas: International Journal of Voluntary and Nonprofit Organizations, vol. 19, $\mathrm{n}^{\mathrm{o}} 1$, pp. 23-42.

WILSON, J. (2000) Volunteering, Annual Review of Sociology, vol. 26, nº 1, pp. 215-240.

WILSON, J. y MUSICK, M. (1997) Who cares? Toward an integrated theory of volunteer work, American Sociological Review, nº 62, pp. 694-713.

WOLFF, N., WEISBROD, B. and BIRD, E. (1993) The supply of volunteer labor: the case of hospitals, Nonprofit Management and Leadership, $\mathrm{n}^{\mathrm{o}}$ 4, pp. 23-45.

WUTHNOW, R. (1995) Learning to Care, New York: Oxford University Press. 